\title{
Low-dose oral chemotherapy and crizotinib for the manifold manifestations of inflammatory myofibroblastic tumor in children
}

\author{
SATISHKUMAR MEENA ${ }^{1}$, RAMYA UPPULURI ${ }^{2}$, HARIKA VARLA ${ }^{2}$, NIKILA \\ RAVICHANDRAN ${ }^{2}$, KESAVAN MELARCODE RAMANAN ${ }^{2}$, Venkateshwaran \\ Vellaichamyswaminathan ${ }^{2}$, RADHAKRISHNAN SATHEESAN ${ }^{1}$, RAJIV LAZARUS \\ PADANKATTI $^{1}$, ANNAPURNESWARI SUBRAMANYAN ${ }^{1}$, ANN KURIAN ${ }^{1}$, Srinivas $\mathrm{S}^{1}$, \\ and REVATHI RAJ ${ }^{3}$
}

${ }^{1}$ Apollo Hospitals Chennai

${ }^{2}$ Apollo Speciality Hospital

${ }^{3}$ APOLLO SPECIALITY HOSPITAL

August 12, 2020

\begin{abstract}
We present our experience in the management of children with inflammatory myofibroblastic tumor, a total of five children with two presenting with superior mediastinal syndrome and three with abdominal disease. We treated all children with a low-dose oral chemotherapy protocol, including prednisolone, 6-mercaptopurine, methotrexate, and celecoxib. Children with the mediastinal disease showed complete response with chemotherapy alone, while the child with sigmoid polyp is in remission with the chemotherapy post complete excision. We commenced two children with abdominal disease on crizotinib due to local recurrence with an adequate response. Long term follow-up and prolonged therapy are essential to sustain remission.
\end{abstract}

\section{Introduction}

Inflammatory myofibroblastic tumor (IMFT) is a rare solid tumor. The prevalence rate is $0.04 \%$ to $0.70 \%$, with a higher incidence in children and young people (1). IMFT was considered a reparative benign postinflammatory condition. However, recent studies have indicated that IMFTs are lesions with intermediary malignant potential due to their tendency to invade tissues, recur locally, and metastasize to distant sites (2).

IMFT can present at any age with a preference for children and adolescents (3). The tumor frequently presents in the abdominopelvic region, lungs, and retroperitoneum. However, it can involve any site of the body (4). The clinical presentation depends on the site of involvement, a palpable abdominal mass being the most frequent physical finding, and fever being the most common symptom. Given the indolent course, the tumor is often diagnosed in an advanced stage (3).

We present data on children diagnosed with IMFT, the varied presentations, challenges during management and follow-up; and the efficacy of an oral out-patient based combination chemotherapy.

\section{Patients and methods}

We performed a retrospective analysis of case records of children less than 18 years of age, diagnosed to have IMFT in the department of pediatric hemato-oncology, from January 2014 till May 2020. The diagnosis was confirmed through morphology and immunohistochemistry (IHC) of an image-guided trucut biopsy of the lesion or review of the excised tissue in those who underwent surgery. The extent of disease required imaging 
with either computed tomography (CT) or Positron emission tomography-computed tomography (PET-CT) as indicated. All cases were discussed in a multidisciplinary tumor board before initiating treatment. Written informed consent was obtained from all families, and the institutional review board has approved the study.

The oral combination chemotherapy regimen included prednisolone at 1 milligram/kilogram/day $(\mathrm{mg} / \mathrm{kg})$ in two to three divided doses, 6-mercaptopurine (6-MP) started at $25 \mathrm{mg}$ once a day in all children and dosage titrated based upon serial absolute neutrophil counts (ANC) such as to maintain an ANC of 750 $1500 / \mathrm{cu} . \mathrm{mm}$, methotrexate at $20 \mathrm{mg} /$ square meter once a week and celecoxib at $50 \mathrm{mg}$ once a day in those between $10-25 \mathrm{kgs}$, and $100 \mathrm{mg}$ once a day in those weighing $>25 \mathrm{~kg}$. All children were monitored for adverse effects with regular assessment for complete blood counts, liver enzymes, and serum creatinine. Response assessment was performed for all children at four weeks of initiating the above chemotherapy. When required, crizotinib was administered at a dose of $280 \mathrm{mg} /$ square meter/day (5).

\section{Results}

Five children were included with the youngest being two years six months and the oldest being 15 years of age, with a prolonged history of fever in all and weight loss in one child. Two children presented with a mediastinal mass and features of superior mediastinal syndrome, and one child each presented with an adrenal mass, midline mass over the bladder and a sigmoid polyp. All five children had localized disease on imaging. Upfront surgery was performed in the three children with abdomen disease, where the polyp was completely excised with negative margins, and $90 \%$ of the tumor was resected in the other two. In both the children with mediastinal mass, image-guided trucut biopsy was performed. Morphology was consistent with IMFT for all five children with predominantly myofibroblastic spindle cells in a background of plasma cells, lymphocytes, and eosinophils. Four of the five children were positive for anaplastic lymphoma kinase (ALK) on IHC.

All children received a combination of chemotherapy, which was well tolerated. One patient (child\#2) had methotrexate-induced toxicity in the form of raised serum bilirubin around three months after initiation of therapy. As he was in remission, we stopped methotrexate and continued 6MP. Child\#5 was kept on the above chemotherapy for three years, given local recurrence on a taper. He is now two years off chemotherapy and is in remission. Child\#2 and 3 were on the medications at the time of this publication.

In both the children with residual abdominal disease post-surgery, localized disease recurrence was noted on imaging, following the initial response. They were both started on Crizotinib, which resulted in complete resolution. Crizotinib continued for one year for child \# 1 and child \# 4 was on the drug for ten months at the time of this publication.

Overall survival in our cohort is $100 \%$, with all children in remission. The longest follow up is five years and the shortest being six months. The details of the five children with their presentations and treatment are elaborated in Table 1. Response evaluations for the children are depicted in Figures 1 and 2. Histopathology and IHC from lung biopsy sample for child\#3 are represented in Figure 3.

\section{Discussion}

Our cohort represents a group of children with IMFT with varied presentations. A low-dose oral out-patient based combination chemotherapy, including prednisolone, 6-mercaptopurine, methotrexate, and celecoxib, was well-tolerated and showed good efficacy. In children with abdominal disease, where complete surgical excision was not possible, recurrence was noted, which responded to targeted therapy with crizotinib.

Diagnosis can be challenging, and a high index of suspicion is essential. IMFTs are characterized by a variably cellular spindle cell proliferation in a myxoid to collagenous stroma with a prominent inflammatory infiltrate composed primarily of plasma cells and lymphocytes, with occasional eosinophils and neutrophils $(4,6)$. The absence of anaplasia, lack of mitotic figures, and the inflammatory background differentiate IMFT from soft tissue sarcomas. IHC may not be definitive because of variable expression and lack of specificity of these markers such as smooth muscle actin, muscle-specific actin, and vimentin. Rearrangements involving the ALK locus on chromosome 2p23 have been documented in approximately $50 \%$ of $\operatorname{IMFTs}(7,8)$. 
Surgery has been known to be the mainstay of treatment. Surgical resection should be recommended for all lesions if not limited by anatomic location or morbidity. Recurrence is rare following complete excision of a solitary lesion, depending upon the anatomical site, from $2 \%$ for tumors confined to the lung to $25 \%$ for extrapulmonary lesions $(9,10)$. We performed surgery for 3 of our cases (child\#1, 2, and 4). In child \#3 and 5, surgery was not offered because of the tumor's difficult anatomic location.

Various adjuvant chemotherapy regimens have been used in the past with limited efficacy $(3,9,11)$. Oral methotrexate and 6-MP have been found to be potentially active chemotherapeutic agents. Several groups have published encouraging results with steroids and non-steroidal anti-inflammatory agents (NSAIDS) with or without concomitant chemotherapy $(12,13)$. Applebaum et al . described the inhibitory effect of NSAIDs through disruption of angiogenesis by interfering with vascular endothelial growth factor signaling via cyclooxygenase 2 (COX-2) inhibition (14).

Crizotinib is a small-molecule tyrosine kinase inhibitor targeting several kinases, including ALK and MET. Recent studies have shown the efficacy of crizotinib in the management of ALK-positive IMFTs (15, 16, 17). In the Children's Oncology Group study, an overall response rate of $86 \%$ was noted with crizotinib in refractory IMFTs, with the most common adverse effect being neutropenia (16).

IMFT requires a multidisciplinary team of pediatric surgeons, pathologists, nursing, and oncologists. Long term follow-up and prolonged therapy are essential to sustain remission. Maximal safe resection followed by oral chemotherapy with low-dose prednisolone, methotrexate, 6-MP, and celecoxib helps achieve remission in two-thirds of the children. Crizotinib is a useful salvage agent in recurrent tumors that are ALK-positive.

Acknowledgments: We would like to acknowledge the contribution of the histopathology department in the management of these children

Conflicts of interest: none

Funding: none

\section{References}

1. Coffin CM, Fletcher JA. Inflammatory myofibroblastic tumor. In: Fletcher CDM, Unni KK, Mertens F, eds. World Health Organization classification of tumors. Pathology and genetics of tumors of soft tissue and bone. Lyon: IARC Press, 2002:91-3.

2. Panagiotopoulos N, Patrini D, Gvinianidze L, et al. Inflammatory myofibroblastic tumor of the lung: a reactive lesion or a true neoplasm? Thorac Dis 2015; 7: 908-11.

3. Dalton BG, Thomas PG, Sharp NE, et al. Inflammatory myofibroblastic tumors in children. J Pediatr Surg. 2016 Apr;51(4):541-4. doi: 10.1016/j.jpedsurg.2015.11.015.

4. Muñoz Moya JE, Alfaro Aguirre MO, Leiva Silva M, Kakarieka Weisskopf E, López Sáez MT. Tumor miofibroblástico inflamatorio: Presentación variable de una misma patología [Inflammatory myofibroblastic tumor: Variable presentation of the same pathology]. Rev Chil Pediatr. 2019;90(3):328-335.

5. Mossé YP, Lim MS, Voss SD, et al. Safety and activity of crizotinib for pediatric patients with refractory solid tumors or anaplastic large-cell lymphoma: a Children's Oncology Group phase 1 consortium study. Lancet Oncol. 2013;14(6):472-480. doi:10.1016/S1470-2045(13)70095-0

6. Gleason BC, Hornick JL. Inflammatory myofibroblastic tumors: where are we now? J Clin Pathol 2008; 61:428-37.

7. Coffin CM, Patel A, Perkins S, et al. ALK1 and p80 expression and chromosomal rearrangements involving 2p23 in an inflammatory myofibroblastic tumor. Mod Pathol 2001; 14:569-76.

8. Griffin CA, Hawkins AL, Dvorak C, et al. Recurrent involvement of 2p23 in inflammatory myofibroblastic tumors. Cancer Res. 1999; 59:2776-80. 
9. Fu GX, Xu CC, Yao NF, Gu JZ, Jiang HL, Han XF. Inflammatory myofibroblastic tumor: A demographic, clinical, and therapeutic study of 92 cases. Math Biosci Eng. 2019;16(6):6794-6804. doi:10.3934/mbe.2019339

10. Shetty SJ, Pereira T, Desai RS. Inflammatory myofibroblastic tumor of the oral cavity: A case report and literature review. J Cancer Res Ther. 2019;15(3):725-728. doi:10.4103/jcrt.JCRT_1044_16

11. Maruyama Y, Fukushima T, Gomi D, et al. Relapsed and unresectable inflammatory myofibroblastic tumor responded to chemotherapy: A case report and review of the literature. Mol Clin Oncol. 2017;7(4):521524. doi:10.3892/mco.2017.1383

12. Tao YL, Wang ZJ, Han JG, et al. Inflammatory myofibroblastic tumor successfully treated with chemotherapy and non-steroidal: A case report: World J Gastroenterol. 2012;18: 7100-7103.

13. Kusunoki-Nakamoto F, Matsukawa T, Tanaka M, et al. Successful treatment of an unresectable inflammatory myofibroblastic tumor of the frontal bone using a cyclooxygenase- 2 inhibitor and methotrexate. Intern Med. 2013; 52: 623-628.

14. Applebaum H, Kieran MW, Cripe TP, et al. The rationale for non-steroidal anti-inflammatory drug therapy for inflammatory myofibroblastic tumors: a Children's Oncology Group study. J Pediatr Surg. 2005; 40: $999-1003$.

15. Theilen TM, Soerensen J, Bochennek K, et al. Crizotinib in ALK+ inflammatory myofibroblastic tumors-Current experience and future perspectives. Pediatr Blood Cancer. 2018;65(4):10.1002/pbc.26920. doi:10.1002/pbc. 26920

16. Mossé YP, Voss SD, Lim MS, et al. Targeting ALK With Crizotinib in Pediatric Anaplastic Large Cell Lymphoma and Inflammatory Myofibroblastic Tumor: A Children's Oncology Group Study. J Clin Oncol. 2017;35(28):3215-3221. doi:10.1200/JCO.2017.73.4830

17. Ogata M, Hatachi Y, Ogata T, Satake H, Imai Y, Yasui H. Effectiveness of Crizotinib for Inflammatory Myofibroblastic Tumor with ALK mutation. Intern Med. 2019;58(7):1029-1032. doi:10.2169/internalmedicine.1640-18

\section{Legends}

Table 1: Patient characteristics, investigations, IHC, treatment, response and follow up

Figure 1: USG abdomen of child\#1 showing a well defined smoothly surfaced spherical mass in the right hilum (a) persistence/recurrence of lesion after 3 months of surgery (b) and disappearance of lesion after 3 months of Crizotinib therapy (c)

Figure 2: Contrast enhanced CT chest of child\#5 showing the mass in upper lobe of right lung with obstruction of the proximal right main bronchus and right upper lobe bronchus

Figure 3: Histopathology and immunohistochemistry for Ki67, ALK, Vimentin and Smooth muscle actin from lung biopsy sample for child\#3

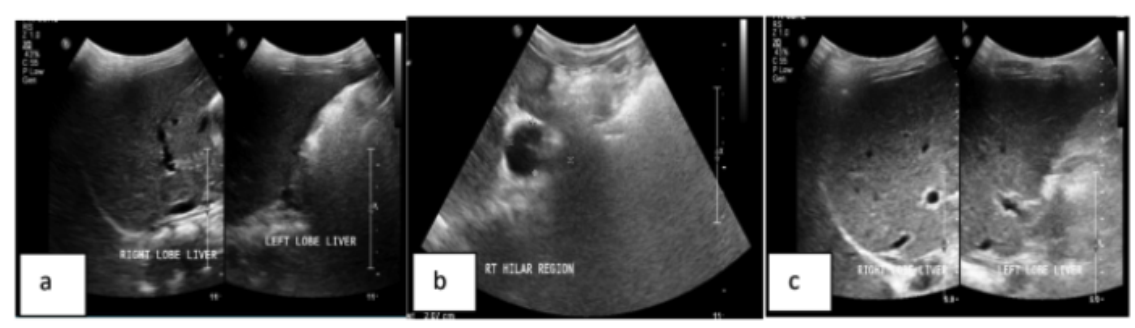



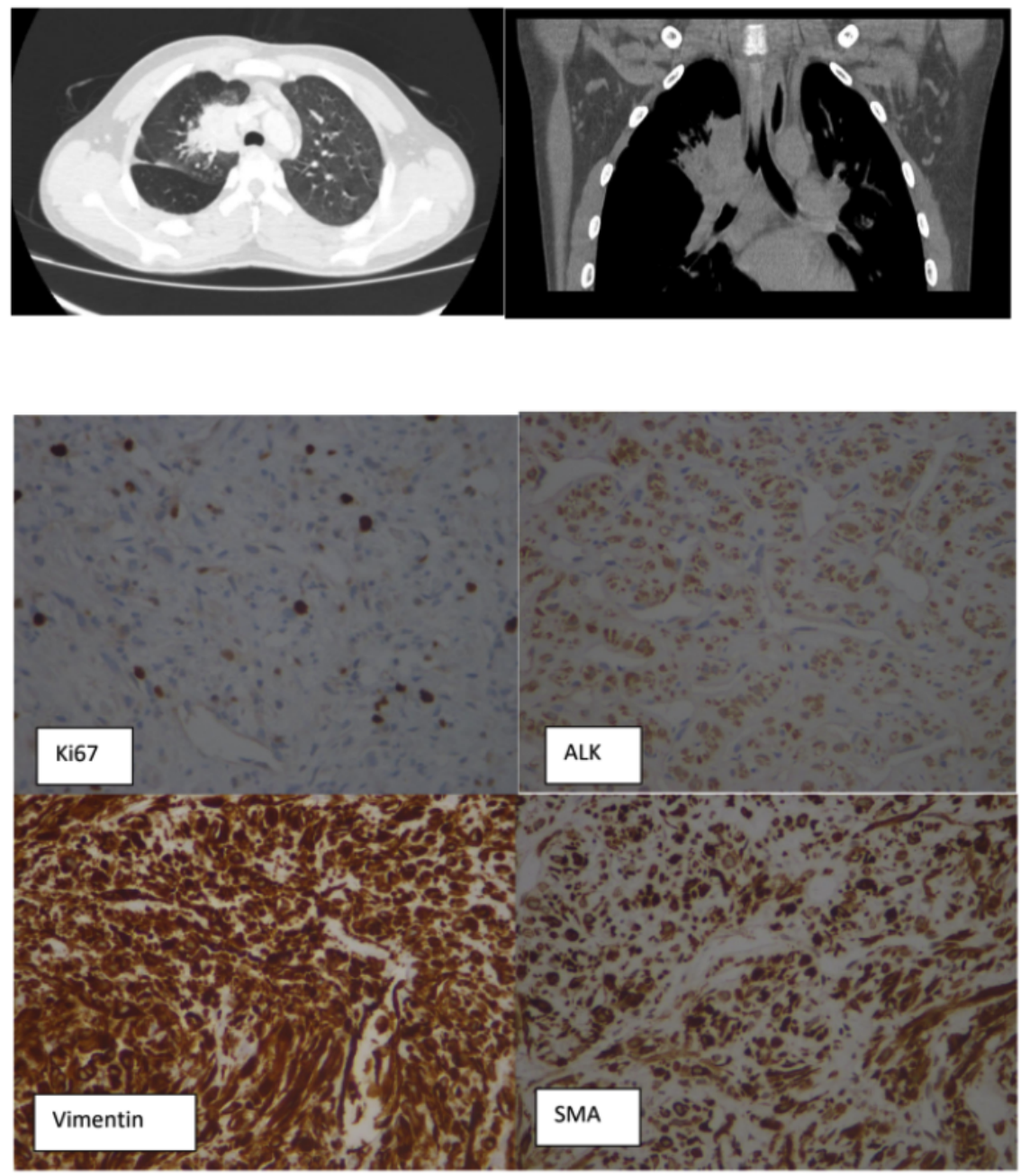

\section{Hosted file}

Table 1 IMFT.docx available at https://authorea.com/users/350426/articles/475267-lowdose-oral-chemotherapy-and-crizotinib-for-the-manifold-manifestations-of-inflammatorymyofibroblastic-tumor-in-children 